\title{
Effects of long-term repeated topical fluoride applications and adhesion promoter on shear bond strengths of orthodontic brackets
}

\author{
Toshiya Endo ${ }^{1}$, Rieko Ishida ${ }^{1}$, Akira Komatsuzaki ${ }^{2}$, Shinya Sanpei ${ }^{3}$, Satoshi Tanaka ${ }^{4}$, \\ Tsuneo Sekimoto ${ }^{4}$
}

Correspondence: Dr. Toshiya Endo

Email: endoto@ngt.ndu.ac.jp

\begin{abstract}
'Orthodontic Dentistry, The Nippon Dental University Niigata Hospital, Niigata, Japan,

${ }^{2}$ Department of Preventive and Community Dentistry, The Nippon Dental University School of Life Dentistry at Niigata, Niigata, Japan,

${ }^{3}$ Pediatric Dentistry, The Nippon Dental University Niigata Hospital, Niigata, Japan,

${ }^{4}$ Department of Pediatric Dentistry, The Nippon Dental University School of Life Dentistry at Niigata, Niigata, Japan
\end{abstract}

\section{ABSTRACT}

Objective: The purpose of this study was to assess the effects of long-term repeated topical application of fluoride before bonding and an adhesion promoter on the bond strength of orthodontic brackets. Materials and Methods: A total of 76 bovine incisors were collected and divided equally into four groups. In group 1, the brackets were bonded without topical fluoride application or adhesion promoter. In group 2, before bonding, the adhesion promoter was applied to nonfluoridated enamel. In group 3, the brackets were bonded without the application of the adhesion promoter to enamel, which had undergone long-term repeated topical fluoride treatments. Teeth in group 4 received the long-term repeated topical applications of fluoride, and the brackets were bonded using the adhesion promoter. All the brackets were bonded using BeautyOrtho Bond self-etching adhesive. The shear bond strength was measured and the bond failure modes were evaluated with the use of the adhesive remnant index (ARI) after debonding. Results: The mean shear bond strength was significantly lower in group 3 than in groups 1,2 , and 4 , and there were no significant differences between the groups except for group 3 . There were significant differences in the distribution of ARI scores between groups 2 and 3 , and between groups 3 and 4. Conclusions: The adhesion promoter can recover the bond strength reduced by the long-term repeated topical applications of fluoride to the prefluoridation level and had a significantly great amount of adhesives left on either fluoridated or nonfluoridated enamel.

Key words: Adhesion promoter,fluoride, orthodontic bracket, shear bond strength

\section{INTRODUCTION}

Topical fluoride products are available in the form of toothpastes, mouthrinses, gels, and varnishes. They have been used, either alone or in combination, to prevent dental caries and white spot lesions in children and adolescents. ${ }^{[1,2]}$ Orthodontic treatments with fixed appliances increase the risk of developing white spots under or around brackets, especially in patients whose oral hygiene status is poor. ${ }^{[3]}$ Fluoride-releasing orthodontic adhesives have been used to solve this and other cariogenic problems during orthodontic treatment. ${ }^{[4,5]}$ Moreover, several methods of topical fluoride application have been proposed at different steps of the orthodontic bonding procedure..$^{[6-13]}$

Some studies have disclosed significantly reduced bond strengths of orthodontic brackets due to

\footnotetext{
How to cite this article: Endo T, Ishida R, Komatsuzaki A, Sanpei S, Tanaka S, Sekimoto T. Effects of long-term repeated topical fluoride applications and adhesion promoter on shear bond strengths of orthodontic brackets. Eur J Dent 2014;8:431-6.

Copyright @ 2014 Dental Investigations Society.

DOI: $10.4103 / 1305-7456.143609$
} 
the topical application of fluoride during the bonding procedure, ${ }^{[7,13]}$ whereas other studies have demonstrated a significant increase or no changes in bond strength. ${ }^{[6,8-12]}$ These studies have evaluated the effect on the bond strength of the short-term topical fluoride application at different steps of the bonding process on the bond strength.

Enamel fluorosis is characterized by an outer hypermineralized, acid-resistant layer and by the formation of more porous enamel with a subsurface hypomineralization. ${ }^{[14]}$ Some researchers have reported that the fluorosed teeth show significant reductions in the bond strength of brackets, when some self-etching adhesive systems were used, compared with the nonfluorosed teeth, ${ }^{[15]}$ but no significant changes when acid-etching adhesive systems were used. ${ }^{[15-18]}$ Some studies have shown that the fluorosed teeth significantly decrease the bracket bond strength in the acid-etching adhesive systems, compared with the nonfluorosed teeth. ${ }^{[18-20]}$ Some investigators have recommended using adhesion promoters to increase the bond strength of the brackets bonded to fluorosed enamel..$^{[17,21]}$

To the best of our knowledge, there were no published studies that have cast light on the effects of the long-term topical application of fluoride and the adhesion promoter on the bond strength of brackets. The purposes of this study were to assess the effects of the long-term repeated topical applications of fluoride before the bonding procedure and the adhesion promoter on the bond strength of orthodontic brackets.

\section{MATERIALS AND METHODS}

The protocol was approved by the Local Committee of Ethics (ECNG-H-129). Sample size was calculated based on a power analysis using $G$ power software version 3 (Heinrich Heine University, Dusseldorf, Germany) for a one-way analysis of variance (ANOVA) at an effect size of 0.4 , an alpha error probability of 0.05 , a power of 0.8 and 4 groups. ${ }^{[22]}$ The power analysis showed that a total of 76 bovine teeth were required and hence it was decided that the sample size in each group was 19 teeth.

A total of 76 bovine incisors were collected and stored in artificial saliva at $37^{\circ} \mathrm{C}$ for $24 \mathrm{~h}$ prior to testing. The criteria for tooth selection included intact buccal enamel with no cracks incidental to extraction and no caries. The teeth were cleansed of soft tissue, and the buccal surface of each tooth crown was polished with 600-grit silicon carbide abrasive paper under the continuous stream water to expose the bonding area and cleansed with a mixture of water and fluoride-free pumice in a rubber prophylactic cup for $10 \mathrm{~s}$. All teeth were divided equally into four groups of 19 teeth each.

Central incisor stainless steel brackets with a 0.018 -inch slot (Victory series; 3M Unitek, Monrovia, CA, USA) were bonded by one operator. The average bracket base area was determined to be $13.23 \mathrm{~mm}^{2}$.

In group 1, each tooth was rinsed with a water spray for $10 \mathrm{~s}$ and dried with an oil-free air drier. BeautyOrtho Bond self-etching primer (Shofu, Kyoto, Japan) was rubbed on the buccal enamel surface for $5 \mathrm{~s}$ and blew off gently with the oil-free air drier for $2 \mathrm{~s}$.

In group 2, each tooth was rinsed and dried the same way as was done in group 1. A thin uniform layer of an adhesion promoter (Shofu) was applied with a brush to the buccal enamel surface, and then BeautyOrtho Bond self-etching primer was rubbed the same way as was done in group 1 . This adhesion promoter was developed for the self-etching adhesive systems according to the manufacture's instruction.

Prior to bracket bonding, teeth in groups 3 and 4 were immersed in a sodium fluoride $(\mathrm{NaF})$ solution (1000 ppm F) for 2 min 3 times a day for a 28-day period. This fluoride application simulated the tooth brushing for 2 min 3 times a day for 1 month using 1000 ppm F-containing toothpaste. The teeth alternated between the NaF solution and artificial saliva. After topical fluoride application, the teeth in group 3 were rinsed, dried and then BeautyOrtho Bond was applied to the buccal enamel surfaces the same way as was done in group 1.

The teeth in group 4 were also rinsed, dried and then the adhesion promoter and BeautyOrtho Bond self-etching primer were applied to the buccal enamel surfaces the same way as was done in group 2 .

After self-etching, BeautyOrtho Bond paste (Shofu) was applied to the bracket base. The bracket was put on the buccal surface of the tooth and pressed firmly into place to squeeze paste from the rim of the bracket base. Excess paste was removed with an explorer before curing. All the brackets were subjected to a constant force of $300-\mathrm{g}$, as measured with a newly developed apparatus (Shofu). The bracket was light-cured with a halogen curing light (Griplight II, SGL-75, Shofu, Japan) for $20 \mathrm{~s}$; $10 \mathrm{~s}$ mesially and $10 \mathrm{~s}$ distally. 
The tooth crown bonded the bracket was embedded in a specimen holder ring with a self-curing acrylic resin and oriented so that the buccal enamel surface would become parallel to, and would project above, the brim of the cylindrical specimen holder ring. All specimen holder rings with the embedded teeth were stored in artificial saliva at $37^{\circ} \mathrm{C}$ for $24 \mathrm{~h}$.

Shear bond strengths were measured on a universal testing machine (Model 5543; Instron Co., MA, USA). The specimen holder rings were arranged in this machine so that a load was applied to the bracket wings with a force in the occlusogingival direction parallel to the buccal enamel surface. The force required to shear off the bracket was recorded in Newtons at a cross-head speed of $1.0 \mathrm{~mm} / \mathrm{min}$. The shear bond strength (MPa) was then calculated by dividing the shear force by the bracket base area.

After each shear bond strength was measured, the bracket base and the enamel surface were coded for each specimen by a person who was not directly involved in this study. These coded bracket bases and enamel surfaces were examined by an investigator (ST), who used a stereomicroscope at $\times 8$ magnification to evaluate the bond failure modes with the adhesive remnant index (ARI). ${ }^{[23]}$ ARI scores ranged from 0 to 3 , with 0 indicating that no adhesive remained on the tooth surface; 1 showing that less than half the adhesive remained on the tooth surface; 2 indicating that more than half of the adhesive remained on the tooth surface, and 3 meaning that all adhesive remnants on the tooth surface had a distinct impression of the bracket base. Two months later, the ARI scores were reexamined by the same investigator (ST) and another investigator (SS) independently. To avoid any examination bias, the investigators examined the coded materials blindly to group. Intra- and inter-examiner Kappa values was 1 and 0.894, respectively, thus demonstrating almost perfect intra- and inter-examiner agreement. ${ }^{[24]}$

Two teeth before bonding and after priming in each group, which had not been tested for shear bond strength, were dehydrated, sputter-coated with gold-palladium and examined under a scanning electron microscope (SEM) (SEM, JSM-6390LA, JEOL Ltd., Tokyo, Japan) at $\times 2700$ magnification.

\section{Statistical analysis}

Statistical analyses were performed using the SPSS for Mac version 17.0J (SPSS Japan Inc., Tokyo, Japan) and Excel-Toukei 2006 for Windows (SSRI, Tokyo, Japan). Means, standard deviations, and ranges of bond strengths were calculated for each of the groups. A two-way ANOVA was used to test the main effects of the fluoride applications and the adhesion promoter on the bond strength. If the two-way ANOVA showed a significant interaction between two variables, a one-way ANOVA and Tukey's test were used to compare the shear bond strengths between the groups. A Chi-square test or Fisher's exact test was performed to determine significant differences in the distribution of ARI scores between the groups. All statistical tests were performed at $P<0.05$ level of significance.

\section{RESULTS}

The two-way ANOVA revealed that there were significant differences in bond strength depending on the application or nonapplication of fluorides $(F=11.350, P=0.001$, Power $=0.914)$ and the use or disuse of the adhesion promoter $(F=16,385$, $P=0.000$, Power $=0.979)$. Furthermore, a significant interaction between these two factors was found.

As shown in Table 1, the mean shear bond strengths of four groups reached $6 \mathrm{MPa}$, which is considered a minimum requirement for clinical use. ${ }^{[25]}$ In group 3, however, the shear bond strength of $3(15.8 \%)$ out of 19 specimens were $<6 \mathrm{MPa}$. The one-way ANOVA and Tukey's test showed that the mean shear bond strength was significantly lower in group 3 than in groups 1, 2, and 4, and that there were no significant differences between the groups except for group 3 [Table 1].

Fisher's exact tests showed significant differences in the distribution of ARI scores between groups 2 and 3 , and between groups 3 and 4, thus demonstrating that the teeth in groups 2 and 4 had a significantly greater amount of adhesive remnants on the enamel surface than those in group 3 [Table 2].

Typical SEM images of the enamel surfaces are shown in Figure 1. Figure 1a shows the furrows created in the process of polishing with the abrasive paper on the enamel surface (group 1). In Figure $1 \mathrm{~b}$ and $\mathrm{d}$, the furrows completely disappear, and exposed enamel crystallites and moderate demineralization are observed on the enamel surface (groups 2 and 4). Figure 1c shows the furrows still remaining and mild demineralization occurring with an etching pattern similar to that created in group 1 (group 3). 


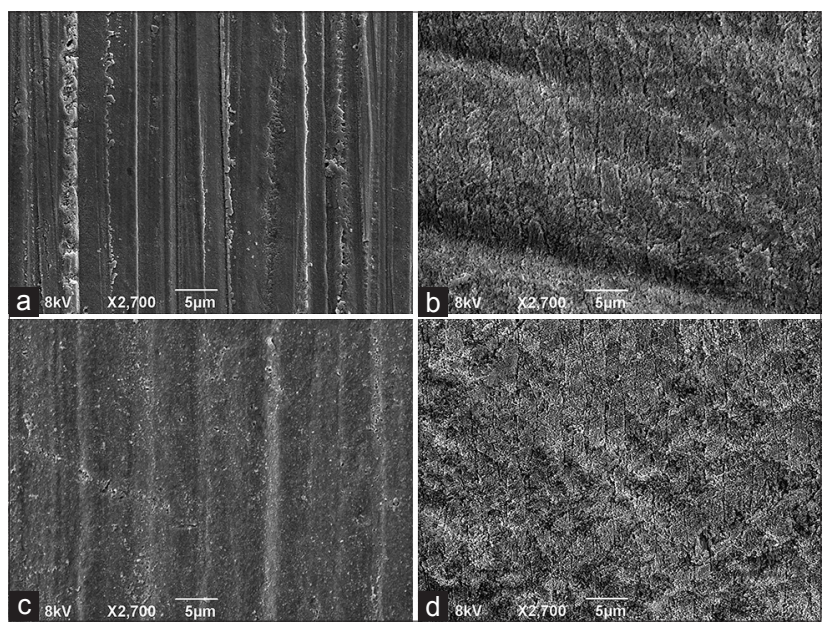

Figure 1: Scanning electron microscope photographs of the enamel surfaces. (a) Without the fluoride and adhesive promoter. (b) Without the fluoride and with the adhesive promoter. (c) With the fluoride and without the adhesive promoter. (d) With the fluoride and adhesive promoter

\begin{tabular}{|c|c|c|c|c|c|c|}
\hline \multirow[t]{2}{*}{ Groups } & \multicolumn{3}{|c|}{$\begin{array}{c}\text { Shear bond } \\
\text { strengths (MPa) }\end{array}$} & \multirow{2}{*}{$\begin{array}{l}\text { ANOVA } \\
P \text { value }\end{array}$} & \multicolumn{2}{|c|}{ Tukey's test } \\
\hline & Mean & SD & Range & & Comparison & $P$ value \\
\hline \multirow[t]{3}{*}{ Group 1} & 22.62 & 6.19 & $9.44-29.88$ & $<0.001^{* * *}$ & 1 vs 2 & $0.846^{\mathrm{NS}}$ \\
\hline & & & & & 1 vs 3 & $<0.001^{* * *}$ \\
\hline & & & & & 1 vs 4 & $0.963^{\mathrm{NS}}$ \\
\hline \multirow[t]{2}{*}{ Group 2} & 24.39 & 5.64 & $7.15-33.05$ & & 2 vs 3 & $<0.001^{* * *}$ \\
\hline & & & & & 2 vs 4 & $0.987^{\mathrm{NS}}$ \\
\hline Group 3 & 13.06 & 6.72 & $1.13-22.35$ & & 3 vs 4 & $<0.001^{* * *}$ \\
\hline
\end{tabular}

Group $423.667 .88 \quad 6.11-35.20$

SD indicates standard deviation, NS: Not significant. ${ }^{* * *} P<0.001$, ANOVA: Analysis of variance

\begin{tabular}{|c|c|c|c|c|c|c|}
\hline \multirow[t]{2}{*}{ Groups } & \multicolumn{4}{|c|}{ ARI scores } & \multicolumn{2}{|c|}{$\begin{array}{c}\text { Chi-square test / Fisher's } \\
\text { exact test }\end{array}$} \\
\hline & 0 & 1 & 2 & 3 & Comparison & $P$ value \\
\hline \multirow[t]{3}{*}{ Group 1} & 0 & 18 & 1 & 0 & 1 vs 2 & $0.180^{\mathrm{NS}}$ \\
\hline & & & & & 1 vs 3 & $>0.999^{N S}$ \\
\hline & & & & & 1 vs 4 & $0.180^{\mathrm{NS}}$ \\
\hline \multirow[t]{2}{*}{ Group 2} & 0 & 14 & 5 & 0 & 2 vs 3 & $0.046^{*}$ \\
\hline & & & & & 2 vs 4 & $>0.999^{N S}$ \\
\hline Group 3 & 0 & 19 & 0 & 0 & 3 vs 4 & $0.046^{*}$ \\
\hline Group 4 & 0 & 14 & 5 & 0 & & \\
\hline
\end{tabular}

NS: Not significant. ${ }^{*} P<0.05$

\section{DISCUSSION}

In this study, the mean shear bond strength of brackets bonded after the long-term repeated topical application of the NaF solution (group 3) decreased significantly, compared with that of the control specimens without topical fluoride application (group 1). Moreover, it was found that the shear bond strengths for three specimens of $19(15.8 \%)$ in group 3 were < $6 \mathrm{MPa}$. Part of these results are consistent with those of Cacciafesta et al..$^{[7]}$ and Meng et al., ${ }^{[13]}$ although there is much difference in the properties of fluorides and etchants as well as the timing of fluoride application between our study and theirs. They used acidulated phosphate fluoride (APF) gels for 4 min once before and after polyacrylic acid-etching, and once after phosphoric acid-etching, respectively. A short-term application of the APF gel in their studies resulted in as significant a reduction in bond strength as our long-term repeated applications of the $\mathrm{NaF}$ solution. This can be explained by the fact that the APF gels are superior to the neutral $\mathrm{NaF}$ solution in depositing fluoride to a great degree in enamel. ${ }^{[26]}$ The studies conducted by Cacciafesta et al. and Meng et al. have suggested that the topical application of fluoride might interfere with the etching effect on the enamel surface and sufficient penetration of the adhesive into the etched enamel, thus resulting in reduced bond strength. ${ }^{[7,13]}$

On the other hand, the results of our study were inconsistent with those of several other studies, which reported that the topical application of various fluoride agents during enamel precleaning, ${ }^{[6,7]}$ before etching, ${ }^{[8,9]}$ during etching, ${ }^{[10,11]}$ and before bonding ${ }^{[12]}$ did not have any adverse effect on the bond strength values of brackets. We used the same fluoride (Durapha, a fluoride varnish containing NaF, Woelm Pharma, Eschwege, West Germany) at the same step of the bonding process as Bryant et al. did, ${ }^{[8]}$ but produced the results different from what they found in regard to bond strength. The probable reason for the discrepancy was that we applied the $\mathrm{NaF}$ solution 3 times a day for 28 days before bonding with the self-etching primer whilst Bryant et al. ${ }^{[8]}$ performed the fluoride application only once before bonding with phosphoric acid. The self-etching primers generally dissolve fluoridated enamel less than phosphoric acid etchants, ${ }^{[27]}$ thus significantly reducing bond strength. It can be speculated that the self-etching adhesive system, as used in this study, has more adverse effects on bond strength than the acid-etching adhesive system. This speculation may be supported by Isci et al., ${ }^{[15]}$ who reported that compared with the nonfluorosed teeth, the fluorosed teeth showed significant reductions in the bond strength of brackets when the self-etching adhesive system was used, but no significant changes when the acid-etching adhesive system was used.

Another probable reason for the different results on the bond strengths between our study and theirs ${ }^{[6-12]}$ might be that we used bovine enamel while they 
conducted the studies in human enamel. Some researchers used bovine teeth, ${ }^{[28]}$ because bovine enamel had the advantages of easy availability and similarities in physical properties and composition to human enamel. ${ }^{[29]}$ Although It has been reported that bovine enamel is a reliable substitute for human enamel in adhesion tests, ${ }^{[30]}$ the bond strength to bovine enamel is $21-44 \%$ lower than human enamel. ${ }^{[29]}$

No literature was found on the association between the long-term repeated topical applications of fluoride and the adhesion promoter in a PubMed search on the Internet. Therefore, the results of our study using the fluoridated teeth were discussed in comparison with those of the study using the fluorosed teeth as experimental materials. ${ }^{[17]}$ Our study found that the application of the adhesion promoter on fluoridated enamel helped restore the bond strength to the prefluoridation level fully. These findings may be consistent with those by Adanir et al. ${ }^{[17]}$ who showed that fluorosis significantly reduced the bond strength of the brackets, while an adhesion promoter trade-named "Enhance LC" (Reliance, Itasca, IL, USA), significantly increased bond strength on fluorosed enamel.

In our study, scanning electron micrographs of the teeth conditioned with the self-etching primer without fluoride application (group 1) showed almost the same enamel surface roughness as those teeth with fluoride application (group 3), although the bond strength was significantly higher in group 1 than in group 3 . Moreover, the SEM showed that the teeth with the application of the adhesion promoter (both groups 2 and 4) had more rough enamel surface than those without the application of the same adhesion promoter (group 1), although there were no significant differences in the bond strength between groups 1, 2, and 4 . These findings may indicate that chemical adhesion is superior to mechanical adhesion in the self-etching adhesive system with an adhesion promoter, thus suggesting that the adhesion promoter on fluoridated enamel with mild surface roughness is capable of restoring the reduced bond strength to the prefluoridation level.

In this study, no significant increase in the mean bond strength value was noted in the nonfluoridated teeth with the adhesion promoter agent (group 2), compared with those without this agent (group 1). These results have been supported by Newman et al., ${ }^{[31]}$ Chung et al., ${ }^{[32]}$ and Vicente et al. ${ }^{[33]}$ who reported that various adhesion promoters, including Enhance LC, All-Bond 2 (Bisco, Schaunburg IL, USA), Megabond, and OrthoSolo (Ormco, Orange, CA, USA), failed to increase significantly the bond strengths for the teeth with normal enamel. On the other hand, some researchers showed that when the rebonded brackets were used, All-Bond 2 improved the bond strengths significantly, ${ }^{[32]}$ while others reported that Enhance failed to improve the rebond strength. ${ }^{[34]}$

Our results showed that either fluoridated or nonfluoridated enamel in groups 2 and 4, when the adhesion promoter was applied, had significantly greater adhesive remnants after debonding than the fluoridated enamel without this promoter application in group 3. These results may be inconsistent with those by Adanir et al., ${ }^{[17]}$ who reported that the fluorosed teeth had greater bond failure at the adhesive-bracket interface, whereas the fluorosed teeth treated with Enhance LC showed bond failure primarily at the enamel-adhesive interface. We found there was only one study which evaluated the effects of fluoride and adhesion promoters on the debonded interface distribution, ${ }^{[17]}$ but the results of the study were contrary to our findings. Therefore, more investigation is needed to clarify the interaction between fluoride agents and adhesive agents.

In this study, the sample size of 19 in each group was computed as a function of the required power level (0.8), the type I error probability (0.05), and the effect size (0.4). This priori power analysis is an efficient method of controlling statistical power and determining the sample size before a study is actually conducted. ${ }^{[22]}$ However, our sample size (19) was not supported by Fox et al., ${ }^{[35]}$ who reported that at least 20 samples should be used to obtain reliable results for bonding evaluations.

\section{CONCLUSIONS}

The long-term repeated topical applications of the sodium fluoride solution reduced the bond strength of the orthodontic brackets bonded to bovine teeth with the self-etching adhesive system. The adhesion promoter used can recover the reduced bond strength to the prefluoridation levels. This promoter had a significantly great amount of adhesives left on either fluoridated or nonfluoridated enamel.

\section{ACKNOWLEDGMENT}

We thank Shofu Inc. for supplying the BeautyOrtho Bond and the adhesion promoter free of charge. 
Endo, et al:: Fluoride, adhesion promoter, and bond strength

\section{REFERENCES}

1. Miller FY, Campus G, Giuliana G, Piscopo MR, Pizzo G. Topical fluoride for preventing dental caries in children and adolescents. Curr Pharm Des 2012;18:5532-41.

2. Chang HS, Walsh LJ, Freer TJ. Enamel demineralization during orthodontic treatment. Aetiology and prevention. Aust Dent J 1997:42:322-7.

3. Tufekci E, Dixon JS, Gunsolley JC, Lindauer SJ. Prevalence of white spot lesions during orthodontic treatment with fixed appliances. Angle Orthod 2011;81:206-10.

4. Endo T, Ozoe R, Shinkai K, Aoyagi M, Kurokawa H, Katoh Y, et al. Shear bond strength of brackets rebonded with a fluoride-releasing and -recharging adhesive system. Angle Orthod 2009;79:564-70.

5. da Silva Fidalgo TK, Pithon MM, do Santos RL, de Alencar NA, Abrahão AC, Maia LC. Influence of topical fluoride application on mechanical properties of orthodontic bonding materials under $\mathrm{pH}$ cycling. Angle Orthod 2012;82:1071-7.

6. Damon PL, Bishara SE, Olsen ME, Jakobsen JR. Effects of fluoride application on shear bond strength of orthodontic brackets. Angle Orthod 1996;66:61-4

7. Cacciafesta V, Sfondrini MF, Calvi D, Scribante A. Effect of fluoride application on shear bond strength of brackets bonded with a resin-modified glass-ionomer. Am J Orthod Dentofacial Orthop 2005;127:580-3; quiz 626.

8. Bryant S, Retief DH, Bradley EL Jr, Denys FR. The effect of topical fluoride treatment on enamel fluoride uptake and the tensile bond strength of an orthodontic bonding resin. Am J Orthod 1985;87:294-302.

9. Wang WN, Sheen DH. The effect of pretreatment with fluoride on the tensile strength of orthodontic bonding. Angle Orthod 1991;61:31-4.

10. Garcia-Godoy F, Hubbard GW, Storey AT. Effect of a fluoridated etching gel on enamel morphology and shear bond strength of orthodontic brackets. Am J Orthod Dentofacial Orthop 1991;100:163-70.

11. Meng CL, Wang WN, Yeh IS. Fluoridated etching on orthodontic bonding. Am J Orthod Dentofacial Orthop 1997;112:259-62.

12. Bishara SE, Chan D, Abadir EA. The effect on the bonding strength of orthodontic brackets of fluoride application after etching. Am J Orthod Dentofacial Orthop 1989;95:259-60.

13. Meng CL, Li CH, Wang WN. Bond strength with APF applied after acid etching. Am J Orthod Dentofacial Orthop 1998;114:510-3.

14. DenBesten PK, Thariani H. Biological mechanisms of fluorosis and level and timing of systemic exposure to fluoride with respect to fluorosis. J Dent Res 1992;71:1238-43.

15. Isci D, Sahin Saglam AM, Alkis H, Elekdag-Turk S, Turk T. Effects of fluorosis on the shear bond strength of orthodontic brackets bonded with a self-etching primer. Eur J Orthod 2011;33:161-6.

16. Ng'ang'a PM, Ogaard B, Cruz R, Chindia ML, Aasrum E. Tensile strength of orthodontic brackets bonded directly to fluorotic and nonfluorotic teeth: an in vitro comparative study. Am J Orthod Dentofacial Orthop 1992;102:244-50.

17. Adanir N, Türkkahraman H, Yalçin Güngör A. Effects of adhesion promoters on the shear bond strengths of orthodontic brackets to fluorosed enamel. Eur J Orthod 2009;31:276-80.

18. Gungor AY, Turkkahraman H, Adanir N, Alkis H. Effects of fluorosis and self etching primers on shear bond strengths of orthodontic brackets. Eur J Dent 2009;3:173-7.

19. Adanir N, Türkkahraman H, Güngör AY. Effects of fluorosis and bleaching on shear bond strengths of orthodontic brackets. Eur J Dent 2007;1:230-5.
20. Silva-Benítez EL, Zavala-Alonso V, Martinez-Castanon GA, Loyola-Rodriguez JP, Patiño-Marin N, Ortega-Pedrajo I, et al. Shear bond strength evaluation of bonded molar tubes on fluorotic molars. Angle Orthod 2013;83:152-7.

21. Noble J, Karaiskos NE, Wiltshire WA. In vivo bonding of orthodontic brackets to fluorosed enamel using an adhesion promotor. Angle Orthod 2008;78:357-60

22. Faul F, Erdfelder E, Lang AG, Buchner A. G*Power 3: A flexible statistical power analysis program for the social, behavioral, and biomedical sciences. Behav Res Methods 2007;39:175-91.

23. Artun J, Bergland S. Clinical trials with crystal growth conditioning as an alternative to acid-etch enamel pretreatment. Am J Orthod 1984;85:333-40

24. Landis JR, Koch GG. The measurement of observer agreement for categorical data. Biometrics 1977;33:159-74

25. Reynolds IR. A review of direct orthodontic bonding. Br J Orthod 1975;2:71-8

26. Zachrisson BU. Fluoride application procedures in orthodontic practice, current concepts. Angle Orthod 1975;45:72-81.

27. Kanemura N, Sano H, Tagami J. Tensile bond strength to and SEM evaluation of ground and intact enamel surfaces. J Dent 1999;27:523-30

28. Sfondrini MF, Cacciafesta V, Scribante A, De Angelis M, Klersy C. Effect of blood contamination on shear bond strength of brackets bonded with conventional and self-etching primers. Am J Orthod Dentofacial Orthop 2004;125:357-60.

29. Oesterle LJ, Shellhart WC, Belanger GK. The use of bovine enamel in bonding studies. Am J Orthod Dentofacial Orthop 1998;114:514-9.

30. Nakamichi I, Iwaku M, Fusayama T. Bovine teeth as possible substitutes in the adhesion test. J Dent Res 1983;62:1076-81.

31. Newman GV, Newman RA, Sun BI, Ha JL, Ozsoylu SA. Adhesion promoters, their effect on the bond strength of metal brackets. Am J Orthod Dentofacial Orthop 1995;108:237-41.

32. Chung $\mathrm{CH}$, Fadem BW, Levitt HL, Mante FK. Effects of two adhesion boosters on the shear bond strength of new and rebonded orthodontic brackets. Am J Orthod Dentofacial Orthop 2000;118:295-9.

33. Vicente A, Bravo LA, Romero M, Ortíz AJ, Canteras M. Effects of 3 adhesion promoters on the shear bond strength of orthodontic brackets: An in-vitro study. Am J Orthod Dentofacial Orthop 2006;129:390-5.

34. Egan FR, Alexander SA, Cartwright GE. Bond strength of rebonded orthodontic brackets. Am J Orthod Dentofacial Orthop 1996;109:64-70.

35. Fox NA, McCabe JF, Buckley JG. A critique of bond strength testing in orthodontics. Br J Orthod 1994;21:33-43.

\begin{tabular}{|l|l|}
\hline \multicolumn{2}{|c|}{ Access this article online } \\
\hline Quick Response Code: & Website: \\
& www.eurjdent.com \\
& Source of Support: Nil. \\
&
\end{tabular}

